\title{
DE LA BIOÉTICA CLÍNICA A LA BIOÉTICA GLOBAL: TREINTA AÑOS DE EVOLUCIÓN
}

\author{
Diego Gracia Guillén*
}

Resumen: Los seres humanos son respetables porque son seres humanos, no porque tengan los mismos valores o compartan las mismas creencias. Una nueva generación de derechos humanos conlleva necesariamente a un nuevo proceso de legitimación de las instituciones políticas. Es el tema de "democracia y bioética". La vida se encuentra amenazada y de ahí emerge la importancia de elaborar una nueva tabla de derechos humanos, los derechos ecológicos y del medio ambiente, los derechos de las colectividades y los derechos de las futuras generaciones.

Los nuevos derechos son globales y por tanto exigen un nuevo tipo de democracia, la llamada democracia global la cual debe ser deliberativa. La bioética es en esencia deliberación y, en ese sentido, debe hacer escuela. La democracia deliberativa podrá ser algún día realidad en la medida que se instale en la sociedad la confrontación plural de ideas.

Palabras clave: Bioética, derechos humanos, democracia, globalización, deliberación

\section{FROM CLINICAL BIOETHICS TO A GLOBAL BIOETHICS: THIRTY YEARS OF EVOLUTION}

\begin{abstract}
Human Beings are respectable because they are Human Beings; not because they have the same worth or share the same beliefs. A new generation of human rights necessitates a new process of legitimization of political institutions. This is the essence of "Democracy and Bioethics". Life as we know it is under threat and from there emerges the importance of formulating a new table of human rights; ecological and environmental rights; the rights of different groups within society and the rights of future generations.

These new rights are global and therefore demand a new type of democracy; it is this call for a global democracy, which must be discussed. Bioethics, in essence is a process of deliberation and discussion and therefore it is fitting that bioethics should provide the arena for discussion. It is important to realize that one day, deliberative democracy could be the reality, and be responsible for establishing a debate which reflects the diversity of ideas within society.
\end{abstract}

Keywords: Bioethics, human rights, democracy, globalization, deliberation

\section{DA BIOÉTICA CLÍNICA AO BIOÉTICA GLOBAL: TRINTA ANOS DA EVOLUÇÃO}

Resumo: Os seres humanos merecem respeito por sua condição intrínseca e não porque tenham os mesmos valores ou compartam as mesmas crenças. Uma nova geração de direitos humanos conduz necessariamente a novo processo de legitimação das instituições políticas. Esse é o tema da "democracia e bioética". A vida encontra-se ameaçada, o que faz emergir a importância em elaborar nova carta de direitos humanos, os direitos ecológicos e do meio ambiente, os direitos das coletividades e os direitos das futuras gerações.

Esses novos direitos são globais e, portanto, exigem novo modelo de democracia, a chamada democracia global que é necessariamente deliberativa. A bioética é, em essência, deliberação e, nesse sentido pode ser guia dessa transformação. A democracia deliberativa poderá ser realidade algum dia na medida em que se instaure na sociedade a confrontação de idéias plurais.

Palavras chave: Bioética, direitos humanos, democracia, globalização, deliberação

\footnotetext{
* Doctor en Medicina. Director del Departamento de Salud Pública e Historia de la Ciencia, Universidad Complutense de Madrid, España.

Correspondencia: dgracia@fes.es
} 


\section{Introducción}

La bioética es un movimiento muy reciente: tiene apenas treinta años de vida. A lo largo de ellos, y con una periodicidad que viene a coincidir con la década, ha ido enfrentando diferentes cuestiones, hasta el punto de que puede hablarse de tres generaciones de problemas, la de los años setenta, la de los ochenta y la de los noventa. Por eso cabe ver el desarrollo de la bioética como una pieza teatral, con un escenario y tres actos, correspondientes a las tres fases por las que ha ido pasando en su desarrollo. De ahí que mi exposición vaya a constar de cuatro partes:

- Primero, el escenario sobre el que hay que situar este movimiento y desde el que adquiere sentido

- Segundo, los problemas propios de la primera década, el movimiento de autogestión del cuerpo y el principio de autonomía.

- En tercer lugar, los problemas bioéticos de segunda generación: las cuestiones relacionadas con la economía, la distribución de recursos y el principio de justicia.

- Y finalmente veremos la tercera generación de problemas bioéticos, todos los relacionados con el fenómeno de la globalización, la protección del medio ambiente y los derechos de futuras generaciones

Como conclusión, intentaré resaltar la importancia que tiene para la sociedad civil la educación en los procedimientos deliberativos.

\section{El escenario: La ética de la vida en la cultura occidental}

El sentido del término bioética viene determinado por las dos palabras griegas que entran en su composición, bios, vida, y éthos, costumbre. Etimológicamente, el término bioética sirve para designar las costumbres que tienen que ver con la vida o el cuidado de la vida. Puede entenderse el término de un segundo modo, dando a sus raíces un sentido más científico. Así, la raíz bíos vendría a designar las ciencias de la vida, y la raíz éthos la ciencia de las costumbres, la ética. De este modo, la bioética sería la parte de la ética que analiza los problemas planteados por las ciencias de la vida, hoy tan acuciantes.

Como puede suponerse, no hay una sola definición del término bioética. Cada grupo lo entiende de un modo distinto, o lo dota de un contenido diferente. Así, por ejemplo, para ciertos autores la bioética debe definirse como la ética de la vida, entendiendo por tal la disciplina que se ocupa de recordar a los seres humanos los deberes sacrosantos que tenemos con la vida, ante el olvido o la negligencia que, según ellos, se produce en este campo. Éstos suelen poner como santo y seña de todo el movimiento, el lema de la "santidad de la vida" o de la "inviolabilidad de la vida humana." Otros, por el contrario, consideran que su objetivo principal es la "calidad de vida", y no la defensa a ultranza de la vida.

Yo no voy a seguir ninguno de esos procedimientos. Pienso que lo más correcto es proceder históricamente, analizando el modo cómo la cultura occidental ha gestionado sus deberes morales para con la vida a lo largo de toda su historia, a fin de que luego veamos las novedades que la bioética ha intentado introducir en este campo y las razones que le han movido a ello.

Nadie pone en duda, ni ha puesto nunca, que la vida es un valor muy importante y que la preservación o conservación de la vida es una obligación moral primaria. Más aún, 
habría que decir que se ha considerado como un valor y un deber de tal importancia, que se ha protegido de una forma que hoy nos parece extrema. Esta forma extrema consiste no sólo en considerar que hay conductas contra la vida que son inmorales y deben ser castigadas con el procedimiento más coactivo que las sociedades tienen, el derecho penal, sino que, además de eso, sin llegar a tales extremos, se ha considerado que los individuos no son quiénes para gestionar su cuerpo y su vida, y que las decisiones sobre ellos deben de tomarlas ciertas personas especialmente cualificadas, como son los médicos o los sacerdotes. Esto es lo que hoy se conoce con el nombre de paternalismo. La gestión tradicional de nuestros deberes para con el cuerpo y la vida ha sido paternalista. ¿Qué quiere decir esto más exactamente?

El paternalismo médico es la teoría que afirma que la gestión del cuerpo del paciente no puede hacerla el propio paciente sino otra persona especialmente cualificada para ello, el médico. No ha sido el único tipo de paternalismo que se ha dado en la cultura occidental. Como principio genérico cabe decir que en ella todas las relaciones sociales importantes se han establecido conforme a ese modelo vertical o paternalista. Los ciudadanos no eran quiénes para intervenir en el gobierno y gestionar la cosa pública, razón por la cual la relación de los gobernantes con sus súbditos era paternalista, en el mejor de los casos, o tiránica, en el peor de ellos. En la época que eufemísticamente se conoce en historia con el nombre de "despotismo ilustrado" se decía aquello de "todo para el pueblo pero sin el pueblo". Esa frase es la quintaesencia de la política paternalista. Y lo mismo cabe decir de todas las demás relaciones sociales, como por ejemplo las de pareja o las que establecen los padres con sus hijos. Éste es el telón de fondo que era necesario tener presente para entender el desarrollo de la bioética. La tesis básica de este telón de fondo es que la gestión del cuerpo, de la sexualidad, de la vida y de la muerte, le ha estado vedada al individuo durante siglos y siglos, quedando a cargo de personas especialmente cualificadas, médicos, gobernantes, sacerdotes, jueces, etc.

\section{Primer acto: Revolución liberal y gestión del cuerpo}

El paternalismo entró en crisis hace ya siglos, con las llamadas revoluciones liberales. Recordemos brevemente lo que sucedió. A partir del año 1517, fecha en que Lutero hace públicas sus noventa y cinco tesis, se inician las guerras modernas de religión entre protestantes y católicos. Duraron más de siglo y medio. El objetivo de esas guerras, en un principio, fue la aniquilación del contrario. No se podía tolerar que alguien defendiera tesis que iban contra la doctrina de la Iglesia de Roma. Esto es lo que en historia se conoce con el nombre de debate sobre la tolerancia. La tesis oficial fue que quienes defendían creencias distintas de las oficiales eran herejes, personas difusoras de doctrinas religiosas erróneas y peligrosas y que, por tanto, debían retractarse o desaparecer. Si a quienes quitan la vida del cuerpo se les condena a muerte, dice Santo Tomás, cuánto más a quienes atentan contra la vida del alma. La intolerancia era considerada una virtud y la tolerancia, un vicio.

Éste fue el punto de partida. Pero el punto de llegada iba a ser muy distinto. En primer lugar, porque ninguno de los dos bandos tuvo la capacidad de exterminar al otro. Según pasaba el tiempo, cada vez era más necesario llegar a un armisticio, a una especie de "coexistencia pacífica"; dicho de otro modo, había que aprender a "tolerar" al discrepante, a pesar de que no tuviera las mismas creencias. 
Es el descubrimiento del llamado "principio de la tolerancia", uno de los grandes temas del siglo XVII: A partir de él se fue elaborando toda la teoría de la "libertad religiosa", entendida ésta como un derecho humano. Los seres humanos son respetables porque son seres humanos, no porque tengan los mismos valores o compartan las mismas creencias. Hay que respetarlos en su diversidad. Ese es el concepto de "libertad de conciencia", que en el siglo XVII cobra cuerpo. Se ha iniciado el mundo moderno, la homogeneidad de valores va poco a poco diluyéndose, y se impone el acuerdo de que los valores de las personas tienen que ser en principio respetados, aunque no coincidan con los nuestros. Es el llamado "derecho a la libertad de conciencia."

En torno a este descubrimiento fundamental se elabora la teoría de los derechos humanos básicos, o derechos personales o subjetivos, el derecho a la vida, a la salud, a la libertad de conciencia y a la propiedad. Locke es el primero que los formula de este modo. La afirmación de estos derechos supone el reconocimiento de la autonomía de los individuos para gobernar su vida y sus asuntos. En primer lugar, los asuntos religiosos. Ya lo hemos visto. Pero muy pronto se llevó este mismo espíritu al tema de los asuntos políticos. De hecho, las revoluciones liberales, a la cabeza de todas la Revolución Francesa, se hizo con la carta de derechos humanos por estandarte. Todos los hombres tienen derecho a intervenir en la elaboración de las leyes y en el gobierno de la cosa pública. Del régimen absolutista o monárquico se pasa al régimen democrático. El soberano ya no es el monarca sino el pueblo.

Pues bien, esa revolución liberal que admite el pluralismo y la autogestión de las creencias religiosas y de las opiniones políticas y que triunfa en los siglos XVII y XVIII, no llega en esa época al espacio de la gestión del cuerpo. Es un fenómeno realmente sorprendente. La revolución liberal no llegó a la medicina. Aquí las decisiones importantes seguían tomándolas los médicos de acuerdo con su sistema de valores, no con el sistema de valores del paciente. Por eso cabe decir que en este espacio de la gestión del cuerpo el paternalismo ha llegado hasta la segunda mitad del siglo XX. Y lo que se dice de la medicina es extensible a las otras dimensiones de la gestión del cuerpo. Así, por ejemplo, la ética sexual, o la ética de la vida y de la muerte han seguido en manos de los teólogos y las iglesias, exactamente igual como sucedía con la ética política antes de las revoluciones liberales. El respeto de la autonomía, es decir, de los sistemas de valores de las personas, no ha llegado a ese ámbito más que en las últimas décadas.

Veamos el caso concreto de la medicina. Si el modelo clásico de relación clínica era el que hemos llamado vertical o monárquico, basado en relaciones de mandato y obediencia, a comienzos del siglo $\mathrm{XX}$ comienzan a introducirse novedades que obligarán a sustituir ese modelo por otro que cabe denominar "oligárquico", en el que el profesional se ve obligado a compartir su poder con otros compañeros, pero sin por eso renunciar a la relación vertical. Sólo en las últimas décadas la relación clínica se ha horizontalizado, y ello por razones que se gestaron fuera del ámbito de la medicina. No han sido los médicos quienes han liderado ese cambio. Todo lo contrario. La medicina ha ido a remolque de otras muchas instituciones sociales, como la política o la familiar, en las que la nivelación de los roles se inició bastante antes. El resultado de este proceso ha sido la inclusión de los pacientes en el proceso de toma de decisiones $\mathrm{y}$, de esta forma, la democratización de las relaciones sanitarias. En el momento en que los usuarios han pasado a participar activamente en el proceso de toma de decisiones, éste ha dejado de ser monárquico u oligárquico, para tornarse en claramente "democrático". 
Esto equivale a decir que en las últimas décadas se ha producido una auténtica revolución o, quizá mejor, que ha tenido lugar la revolución liberal en un nuevo espacio, que ya no es el religioso ni el político, sino el de la gestión del cuerpo. Como en los dos casos anteriores, se trata de superar el paternalismo y considerar a los individuos autónomos y libres para tomar sus propias decisiones; decisiones sobre sus creencias religiosas, sobre sus opciones políticas y sobre la gestión de su cuerpo y de su sexualidad, de su vida y de su muerte. Mientras los individuos sean adultos y capaces, mientras no se trate de niños o de incapaces, nadie tiene derecho a prohibir la autogestión del cuerpo y de la vida de cada uno, de acuerdo con su propio sistema de valores y de creencias. Frente al paternalismo, la autonomía de los ciudadanos en el campo de la gestión del cuerpo, de la sexualidad, de la vida y de la muerte. Esto se produce sobre todo a partir de los años sesenta. De ahí la importancia que adquieren los movimientos a favor de los derechos civiles de aquellos colectivos sociales que no los disfrutaban en plenitud, afroamericanos, mujeres, enfermos, grupos gay, etc. En el caso concreto de la medicina, la aparición de los códigos de derechos de los enfermos y todo el tema del consentimiento informado obedecen a esta mentalidad, que toma cuerpo en la década de los años sesenta y empieza a dar sus frutos maduros en los años setenta.

\section{Segundo acto: Justicia social y distribución equitativa de recursos en salud}

Los años setenta trajeron novedades importantes que obligaron a plantear nuevos problemas. La década se abrió con una famosa crisis económica, la crisis de 1973, la primera crisis de recursos en la historia de la humanidad. Ella ha sido considerada en múltiples ocasiones como el final de la economía keynesiana y el comienzo del declive del Estado de bienestar. Los sistemas de seguro público empezaron a tener problemas graves de financiación, lo que a su vez planteó cuestiones hasta entonces prácticamente desconocidas en ámbitos como el de los seguros obligatorios de asistencia sanitaria, en especial el fenómeno de la explosión de costes y los criterios de distribución de recursos escasos.

Las bases teóricas del Estado de bienestar se instalaron, como es bien sabido, a partir de las revoluciones sociales de la segunda mitad del siglo XIX. El resultado de esas luchas fue la proclamación de una segunda carta de derechos humanos, los llamados derechos económicos, sociales y culturales, entre los que estaba el derecho a la asistencia sanitaria. Ése es el origen de los seguros públicos y obligatorios de enfermedad, que fueron apareciendo poco a poco en la práctica totalidad de los países europeos. Tras la revolución liberal, cuyo estandarte fueron los derechos civiles y políticos, ahora se había producido otra, en la cual las reivindicaciones tenían un carácter más material y tangible. Ya no se trataba de conquistar la libertad frente a las leyes serviles o a los caprichos del monarca y la nobleza, sino de gozar de las condiciones necesarias y suficientes para que esa libertad pudiera ser ejercida realmente. De ahí que los movimientos socialistas esgrimieran siempre la tesis de que sin los derechos económicos, sociales y culturales, la conquista de los derechos civiles y políticos tenía un carácter más formal que real. Los estratos más desprotegidos de la sociedad seguían sufriendo, a pesar de las revoluciones liberales, las consecuencias de la ignorancia, de la marginación y siendo objeto de abuso por parte de los demás miembros de la sociedad. Sólo la puesta en práctica de esa segunda tabla de derechos humanos, podía convertir en realidad 
lo que hasta entonces había sido sólo un sueño, la igualdad básica de oportunidades entre todos los miembros de una sociedad o un Estado.

Adviértase lo que esto suponía. De lo que se trataba era de reformular como obligaciones perfectas o de justicia, muchas de las que hasta entonces habían sido consideradas obligaciones imperfectas o de beneficencia. Dicho de otro modo, se trataba de acabar con el viejo paternalismo, haciendo pasar esos deberes a un nuevo concepto que hubo que crear entonces, el de "justicia social". "Del paternalismo a la justicia social": ése fue el grito de los revolucionarios de 1848 y de los movimientos sociales posteriores a tal fecha. Muchos de los deberes que el Estado liberal consideró privados o de beneficencia, debían verse como públicos o de justicia. Por tanto, no eran deberes imperfectos, o deberes no correlativos a derechos, sino muy al contrario, deberes generados por los derechos de las demás personas. De ahí que, por ejemplo, la asistencia sanitaria pasara de verse como una obligación privada de beneficencia a considerarse un deber público de justicia, de lo que entonces empezó a llamarse justicia social. Por eso el Estado se vio en la obligación de procurar una asistencia mínima decente o decorosa a todos, y a todos por igual.

Pues bien, esto que comenzó a gestarse a mediados del siglo XIX y que fue tomando cuerpo, especialmente en los países europeos, a lo largo del siglo XX, muy en particular en las décadas posteriores a la Segunda Guerra Mundial, entró en crisis en torno a 1973. Hasta entonces todo había ido bien, excesivamente bien. El concepto de justicia social funcionaba sin grandes problemas y el Estado social de mercado parecía sólidamente establecido en la mayoría de los países occidentales.

La explosión de costes y la aparición de números rojos en las cuentas de prácticamente la totalidad de los sistemas de seguro público fue un poderoso toque de atención al conjunto de la sociedad. Era necesario analizar con más detalle el tema de la justicia, el concepto de justicia social. ¿Cuáles eran, realmente, las obligaciones del Estado en este campo? ¿Eran absolutas? ¿Eran totales? ¿Qué debía entenderse por justicia social? Ese fue el gran tema de la segunda mitad de los años setenta y sobre todo de los ochenta, y fue también la gran novedad en los debates de la bioética a lo largo de su segunda década de existencia. De fijar la atención en el tema de la autonomía pasa a ponerse ahora el énfasis en el de la justicia.

Recordemos algunas fechas muy significativas. El año 1971 aparece el gran libro de John Rawls, A Theory of Justice(1), probablemente el de mayor importancia en esta materia a todo lo largo de la centuria. Unos años después, en 1974, le contesta Robert Nozick con Anarchy, State, and Utopia (2), y a partir de ese momento se inicia un debate que ha durado no menos de una década. No hay por qué seguir aquí su desarrollo en detalle. Sí conviene recordar que ese debate general repercutió inmediatamente en bioética, donde el tema de la justicia ocupó y preocupó de modo muy importante a todo lo largo de la década de los años ochenta. Es significativo a tal respecto, por ejemplo, que Norman Daniels, uno de los bioeticistas más significativos en este campo, empezara a aplicar las teorías de Rawls a la asistencia sanitaria en torno a 1980, y que sus dos libros más importantes, Just Health Care(3) y Am I My Parents 'Keeper?(4), se publicaran los años 1985 y 1988.

Del interés por la autonomía a la preocupación por la justicia. Son dos dimensiones de la vida moral que se exigen mutuamente, pero que también entran en permanente conflicto. Sin una justicia que asegure la igualdad básica de oportunidades de 
todos en la vida social, la autonomía se vuelve retórica. De ahí que fuera necesario relegitimar el Estado liberal mediante la adopción de los derechos económicos, sociales y culturales. Y de ahí que en bioética se diera un fenómeno muy similar, y de la defensa de la autonomía de los pacientes se pasara pronto a la preocupación por la justicia sanitaria. Fue el segundo escenario, el segundo acto de esta representación. Pero la cosa no acaba aquí. Porque hay, al menos, otro más, el tercero, que ha ido cobrando progresiva importancia a lo largo de estos últimos años. Veamos en qué consiste.

\section{Tercer acto: Globalización, medio ambiente y futuras generaciones}

Los años noventa han aportado su propia novedad. El término que mejor la define es, quizá, el de "globalización". Vivimos en la aldea global. Las comunicaciones permiten saber lo que sucede en cualquier lugar del planeta en tiempo real y desplazarse físicamente allí en muy pocas horas. Las fronteras de los Estados nacionales se han quedado pequeñas y los problemas son cada vez más globales, afectan a todos. El mercado de capitales y el comercio se han internacionalizado como nunca antes. De ahí que cada vez se haga más necesario pensar en términos globales. Hay que globalizar la economía, porque sólo así será posible conseguir lo que ahora se propone como objetivo fundamental, el "desarrollo sostenible" de todos, frente al desarrollo insostenible del primer mundo y al subdesarrollo, también insostenible, del tercero. Sin desarrollo sostenible no podremos conservar el medio ambiente ni asegurar un futuro digno a las próximas generaciones. Se está produciendo una nueva revolución que, como las dos anteriores tiene como consecuencia el surgimiento de una nueva tabla de derechos humanos, los derechos del medio ambiente y los derechos de futuras generaciones. Una vez más, cuestiones que eran consideradas de gestión privada pasan a convertirse en deberes públicos o de justicia. Sólo así seremos capaces de proteger la vida, el presente y el futuro de la vida. He aquí el nuevo frente que se le ha abierto a la bioética.

Esto, a su vez, obliga a cuestionar las estructuras políticas vigentes. Una nueva generación de derechos humanos conlleva necesariamente un nuevo proceso de legitimación de las instituciones políticas. Es el tema de "democracia y bioética". No es la primera vez que esto sucede. Cada generación de derechos humanos ha tenido por objeto legitimar al poder político. Los derechos civiles y políticos legitimaron el Estado liberal frente al absolutismo propio del antiguo régimen. Lo que se empezó a decir entonces fue que las leyes emanadas del Estado absolutista eran legales pero no legítimas. Por eso hubo que instaurar los parlamentos democráticos. Algo similar sucedió a partir de 1948. La aparición de los derechos económicos, sociales y culturales tuvo por objeto dotar de nueva legitimidad a los Estados, haciéndoles pasar de meros Estados liberales a Estados sociales. No parecía fácil ir en el futuro más allá de ese punto. ¿Cabía imaginar nuevos procesos de legitimación? Las luchas entre el Este y el Oeste que salpicaron la historia de Occidente desde 1848, y sobre todo desde 1917 hasta la caída del muro de Berlín, el año 1989, tenía que conducir necesariamente a la reforma del Estado liberal y a la aparición del Estado social. Pero más allá de éste no era posible concebir ningún otro. La historia parecía terminar ahí. Y sin embargo, poco a poco se ha ido abriendo paso un nuevo horizonte de problemas. La tensión entre el Este y el Oeste ha ido difuminándose hasta desaparecer, y una nueva 
ha surgido, ésta entre el Norte y el Sur. Ahora el conflicto se plantea entre los países desarrollados y aquellos otros que, eufemísticamente, se denominan en vías de desarrollo. Es la confrontación de la vida, de la supervivencia presente y futura y de la calidad de vida. No se trata sólo de la vida humana sino de la vida en general. La vida está amenazada. Y está amenazada, precisamente, por el desarrollo insostenible de los países del llamado Primer Mundo y por el subdesarrollo, también insostenible, de los del Tercero. De ahí la importancia de elaborar una nueva tabla de derechos humanos, los derechos ecológicos y del medio ambiente, los derechos de las colectividades y los derechos de futuras generaciones.

El problema de estos derechos es que no son individuales sino colectivos y que, por ello mismo, no pueden gestionarse más que colectivamente, globalmente. En la primera generación el marco de referencia era el individuo y en la segunda, el Estado. Ahora las fronteras del Estado resultan insuficientes o, mejor, inútiles, cuando no perjudiciales. Los nuevos derechos son globales y exigen, pues, un nuevo tipo de democracia, la llamada democracia global. La tesis que se va poco a poco imponiendo es que el viejo Estado nacional surgido tras la paz de Westfalia, en pleno siglo XVII, toca a su fin y que estamos entrando en una nueva época en que todo, incluidas la política y la ética, habrán de ser globales o no serán nada.

¿Qué es globalizar? Por lo pronto, romper las fronteras nacionales y permitir que todo lo que sucede en el globo terráqueo se haga presente y se viva como propio por cualquier miembro de la comunidad humana. La globalización de las comunicaciones permite ya estar al tanto de las noticias de todo el mundo, poder seguirlas en tiempo real $\mathrm{y}$, de ese modo, sentirlas como propias. Ahora todo afecta a todos. Esto es obvio en el orden informativo y de las comunicaciones. Pero sucede también en otros muchos ámbitos. Pensemos, por ejemplo, en el financiero. Los mercados de capitales se han globalizado, y cualquier pequeño ahorrador del más remoto país puede invertir su dinero en la bolsa de cualquier otra parte del mundo. Lo mismo les sucede a las mercancías. Por vez primera cabe hablar de un mercado mundial. El acero que se consume en Barcelona puede haberse producido en Japón o en Corea y las fresas que comemos pueden ser chilenas.

Esta globalización económica plantea inmediatamente problemas políticos. Ahora, por ejemplo, la liberalización de las economías se convierte en un principio sacrosanto. Se trata de algo tan revolucionario como lo que sucedió en la época de Adam Smith. Si entonces se luchó contra los monopolios mercantiles, ahora se está derribando otro tipo de monopolios mucho más poderosos, los Estados nacionales. La globalización económica exige un nuevo tipo de Estado, con una soberanía, digamos, "limitada".

Y todo esto conlleva una ética. Por primera vez somos conscientes de que nuestras acciones afectan al conjunto de todos los seres humanos, no sólo presentes sino futuros. El principio de universalización formulado por Kant adquiere así un nuevo sentido, imposible de percibir a la altura del siglo XVIII, la época en que Kant vivió. No se trata ya de hacer una pirueta mental para comprobar si el móvil de nuestra voluntad podría convertirse en ley en una sociedad de seres humanos dignos. Ahora no hacen falta esos ejercicios de imaginación, porque por vez primera en la historia tenemos la posibilidad de dar voz a todos aquéllos que puedan verse afectados por el acto o la norma en cuestión. Todos tenemos claro que las 
decisiones que se toman en Washington o en Bruselas afectan a muchas más personas que las que habitan en el interior de las fronteras de Estados Unidos o de la Unión Europea. Muchas de esas decisiones, la mayoría, afectan grande y gravemente a los países del Tercer mundo. Ahora bien, si esto es así, ¿deberían ser tenidos en cuenta sus intereses y no sólo los de los habitantes de esas naciones o zonas? Evidentemente, sí. Y ello por la misma razón por la que en los siglos XVII y XVIII se concedió voz y voto a todos los ciudadanos de un país. La tesis es que todos ellos tenían derecho a intervenir en la formulación de las leyes, precisamente porque eran los depositarios de la soberanía; eran soberanos, el pueblo soberano. Ahora bien, ¿no cabe decir por lo mismo que todos los afectados por una norma, por más que habiten fuera de las fronteras nacionales, deberían tener hoy la capacidad de debatirla e intervenir en su aprobación o reprobación? Y si esto es así, ¿no habría que hablar de algo así como de una soberanía global?

En ética caben pocas dudas a este respecto. El problema de nuestras democracias es que son poco democráticas, es decir, poco representativas, o mejor, poco participativas y poco deliberativas. Dicho de otro modo, el problema de nuestras democracias es que poseen un grave defecto de legitimación moral. ¿Cómo enjuagarlo? Caben muchas posibilidades. Uno se imagina que a través de las nuevas redes de telecomunicación tiene que ser posible abrir la vida política a la participación de todos los interesados en algo, y que de ese modo puede suplirse este defecto crónico de nuestros sistemas políticos. En el tiempo de la Revolución francesa tenía sentido que las provincias tuvieran que elegir sus parlamentarios, para que fueran a París y los representaran. Ese fue el origen de la llamada democracia representativa. Pero hoy, con los nuevos sistemas de comunicación, parece que las cosas debían organizarse de otro modo.

Quienes probablemente pondrán más objeciones a este tipo de razonamientos serán, sin duda, los políticos. Del mismo modo que en el antiguo régimen había unos profesionales de la política que eran los nobles, a partir de las revoluciones liberales surgen otros, que son los 1lamados políticos a secas, los políticos profesionales. Ellos están convencidos que si bien el pueblo es soberano, no sabe gobernar. Los que saben gobernar son ellos. De ahí que la democracia se legitime a través de las urnas, pero dejando claro que en éstas no pueden elegirse más que a políticos profesionales. Lo que el ciudadano hace es elegir entre ellos, nada más. El objetivo de las votaciones no es otro que elegir a los gobernantes entre los distintos miembros de la clase política. Los políticos profesionales tienen bien claro que quienes tienen que gobernar son ellos y no el pueblo. De ahí la alergia que profesan a los sistemas o procedimientos asamblearios. La democracia tiene que ser representativa, no participativa.

El problema es si este punto de vista sigue siendo válido hoy y, sobre todo, si lo va a seguir siendo en el futuro. Dicho de otro modo, la cuestión está en saber si los sistemas representativos son los adecuados para la instauración de una verdadera democracia global.

La opinión que empieza a cundir es que no. Hay que ir más allá de los sistemas representativos, hacia otros básicamente participativos y deliberativos. No es que no tenga que haber políticos; es que deben adquirir un nuevo estilo. Lo que quizá esté a punto de desaparecer es el tipo de político surgido de la Revolución francesa.

La cuestión está entonces en describir con alguna precisión qué se entiende por democracia participativa y deliberativa, o en 
qué han de consistir ambos procedimientos, la participación y la deliberación. La respuesta no es fácil. Basta hojear la abundantísima literatura hoy existente en torno a la democracia deliberativa, para darse cuenta de ello. Pero esa misma producción bibliográfica, surgida en las dos o tres últimas décadas, es a su vez un excelente indicador de lo que está sucediendo en el interior de la ciencia política.

La clave está en el término "deliberación". Aristóteles dejó dicho que la deliberación es el método de la racionalidad práctica y, por ende, el propio de las decisiones técnicas o artísticas, así como de las éticas y las políticas. Se delibera para tomar decisiones, y las decisiones son siempre y necesariamente concretas. Aquí, pues, no valen los juicios universales. Si queremos que nuestras decisiones sean correctas, habremos de tener en cuenta también las circunstancias del acto y las consecuencias previsibles. Las decisiones concretas no pueden tomarse en abstracto. Un capitán de barco no puede guiarse sólo por los principios generales que se explican en los libros de náutica, sino que, además, deberá tener en cuenta las circunstancias concretas en que se encuentra la mar y su barco y que, por tanto, han de ser tenidas en cuenta a la hora de tomar la decisión, si es que pretende que ésta sea prudente y razonable. Para conseguir esto último es para lo que deberá deliberar. La deliberación es el proceso intelectual de ponderación de los factores que deben ser tenidos en cuenta en un proceso razonable de toma de decisiones. Decimos razonable y no racional, porque nunca seremos capaces de incluir todas las circunstancias de una situación, y menos aún de prever todas las consecuencias del acto. Esto es sencillamente imposible. La mente humana no es nunca capaz de agotar la realidad. De lo que cabe concluir que las decisiones concretas no pueden aspirar nunca a la inerrabilidad o infalibilidad. Sus juicios no son como los del álgebra o la trigonometría.
Deben de ser razonables, pero no serán nunca completamente racionales. Y ello por más de un motivo. En primer lugar porque, como acabamos de decir, nunca tienen carácter apodíctico. Y en segundo, porque en esas decisiones juegan un papel importante no sólo las razones sino también otros factores que no son racionales o que, al menos no son completamente racionales, como los sentimientos, los valores, las creencias, etc. No, el razonamiento práctico no es apodíctico o demostrativo. Lo cual permite entender que ante un mismo hecho puedan tomarse distintas decisiones, todas ellas razonables y prudentes. La prudencia no debe confundirse con el consenso, y menos con la unanimidad.

La deliberación es, en consecuencia, un método de conocimiento, un procedimiento intelectual, cuyo objetivo es la toma de decisiones, y de decisiones prudentes. Se delibera dando razones y escuchando las razones de los demás, en el convencimiento de que nadie está en posesión de toda la verdad, precisamente porque, como ya hemos dicho, la realidad siempre nos supera y cualquier acontecimiento tiene más facetas de todas las que nosotros podamos tener en cuenta. El propio proceso de formación profesional es ya un sesgo para el análisis de la realidad. La formación nos hace sensibles a ciertos rasgos de las cosas, a la vez que deja en la penumbra otras. Un médico, un pintor y un donjuán perciben cosas distintas ante un cuerpo de mujer. Y un banquero, un profesor de arte y un constructor perciben cosas distintas ante el espectáculo que les ofrece una catedral gótica.

Todo lo que forma, deforma. Y todo lo que descubre, encubre. El dirigir la mirada hacia algo y verlo con claridad, exige siempre dejar en la penumbra otros aspectos de la cosa. Nunca hay una claridad total. En este mundo no hay una luz que no genere, ella misma, sombras. Como dijo y escribió Ortega, la 
claridad total es característica que sólo puede predicarse de Dios. Por eso no existe la verdad "total". El término griego para verdad es alétheia, que significa des-cubrimiento o desvelación. Se trata de correr el velo que oculta a las cosas, descubrir sus secretos. Pero esa desvelación, por lo dicho, es siempre parcial. De ahí que no sea nunca del todo verdadera. Deliberamos para buscar la verdad, para acercarnos a ella, pero siendo conscientes de que nunca llegaremos a poseerla plenamente. Por eso las otras perspectivas, los otros puntos de vista nos son necesarios. Se delibera con los otros, con las otras personas, para conocer sus puntos de vista sobre la cosa y de ese modo enriquecer nuestro razonamiento con nuevas perspectivas. Cuantas más perspectivas seamos capaces de integrar, más fácil será que nuestra decisión merezca los calificativos de prudente y correcta.

Tras lo dicho, tendría perfecto sentido concluir que las decisiones sociales y políticas deberían tomarse tras un amplio proceso de deliberación, en el que intervinieran la totalidad de los afectados por ellas. Sería la manera de legitimar moralmente esas decisiones, de hacerlas realmente justas, válidas, legítimas. De ahí la importancia que hoy tiene en filosofía política el concepto de democracia deliberativa. La democracia global debe ser deliberativa.

A esto responden los políticos diciendo que se trata de una propuesta puramente ideal y, por tanto, impracticable. ¿Sabe de hecho la gente deliberar? Es indudable que la deliberación tiene unos requisitos: es preciso partir del respeto al otro, a la diferencia, así como saber argumentar, saber dar razones de los propios puntos de vista y ser capaz de prestar atención a las razones de los demás. Por eso Aristóteles incluyó a la prudencia entre las virtudes dianoéticas o intelectuales. Ahora bien, $¿$ sabe hacer esto la gente? Y aun en el caso de que supiera, ¿estaría dispuesta a hacerlo?
No es éste el momento de llegar al fondo de estas cuestiones. Pero sí conviene advertir que esto es lo que está planteando en toda su crudeza el tema de la pedagogía de la deliberación, o de la educación deliberativa. Hay que educar en la deliberación a los niños ya desde la escuela primaria. Esa es la propuesta que una de las más agudas representantes de la democracia deliberativa, Amy Gutmann, ha hecho en su espléndido libro Democratic education(5), recientemente traducido al español. La deliberación no es sólo una metodología; es también una pedagogía, una ética y hasta una ascética.

Y aquí entra en juego la bioética. Porque la ética en general, y la bioética muy en particular, tienen y no pueden no tener por método la deliberación. La bioética es deliberativa. O mejor aún, la bioética es, debe de ser, tiene que ser una escuela de deliberación. Ése es su objetivo. Esa deliberación tuvo por objeto en la década de los años setenta el nivel que podemos denominar "micro", el de la toma de decisiones en torno al propio cuerpo. En la década de los setenta subió un peldaño y se situó en el nivel "meso", el de las decisiones institucionales y estructurales. Y en los años noventa ha ampliado aún más sus horizontes, abarcando también el nivel "macro", el propio de la ética global. Son tres estratos de un mismo proceso, el proceso deliberativo: el estrato personal, el institucional y el global. Mi tesis es que se hallan internamente articulados entre sí, de tal modo que el primero de ellos conduce necesariamente al segundo, y éste al tercero y que, por ende, deben verse como momentos de un todo indisoluble.

¿Qué concluir de todo esto? A mi modo de ver, dos cosas. Primera, que la bioética es cada vez más consciente de que su método es la deliberación. Y segunda, que el ejercicio de su propia metodología la está llevando a enfrentar problemas cada vez más globales, 
hasta el punto de que de ser una disciplina fundamentalmente clínica está pasando paulatinamente a convertirse en un instrumento de análisis social, institucional y político. Eso es lo que hace que cada vez se la vea menos como una ética profesional y más como una ética general, interesada tanto por las dimensiones personales como por las institucionales y globales. Hace ahora más de diez años, en 1989, escribí, en el prólogo de mis Fundamentos de bioética, estas líneas: "Si en otros tiempos la medicina monopolizó las ciencias de la vida, hoy eso no es así y, por tanto, sería un error reducir el ámbito de la bioética al de la ética médica, o convertirla en mera deontología profesional. Se trata, a mi parecer, de mucho más, de la ética civil propia de las sociedades occidentales en estas tortuosas postrimerías del segundo milenio." Hoy, ya doblado el cabo del nuevo milenio, no sabría decir otra cosa.

\section{Conclusión: Por una sociedad deliberativa}

El reto de los años setenta fue la reivindicación de los derechos civiles de los enfermos, tanto somáticos como mentales. En los ochenta el reto pasó de los derechos individuales a los sociales, y el debate giró en torno a los temas de justicia sanitaria. En un cierto momento pudo parecer que no podía irse más allá. Pero la década de los noventa nos ha convencido a todos que aún era necesario ampliar el horizonte y plantearse de frente otros nuevos derechos relativos a la vida y a su gestión, que ya no son individuales ni sociales, sino globales. Se trata de los derechos de la vida en general y, en consecuencia, de los ecosistemas, de la vida humana actual en su totalidad, y de las futuras generaciones. Estos derechos no pueden gestionarse ni individual ni socialmente, es decir, dentro de los límites de las nacionalidades clásicas. Las naciones muestran siempre una gran insensibilidad ante los problemas globales, aunque sólo sea porque, como su nombre indica, se definen por el nacimiento y, por tanto, por la pertenencia al grupo familiar y étnico, al grupo de los próximos, no al de los lejanos. Con los próximos se tienen vínculos afectivos y emocionales que no se dan con los lejanos. Y eso acaba teniendo consecuencias morales de primera categoría. "Ojos que no ven, corazón que no siente", dice la sabiduría popular. Los emocionalmente lejanos difícilmente se nos convierten en perentorio problema moral. De ahí la importancia de aprender a pensar y sentir globalmente. Los derechos sobre el medio ambiente, la búsqueda de un desarrollo sostenible, más allá del desarrollo insostenible del primer mundo y del subdesarrollo, también insostenible, del tercero, y los derechos de las futuras generaciones, no pueden gestionarse más que globalmente.

De ahí la importancia de que la globalización pase de ser meramente mercantil y financiera a convertirse en política. Ese es el objetivo de todo el amplio movimiento teórico en torno a las democracias participativas y deliberativas. Por supuesto, hoy por hoy se trata de meras teorías. Y para muchos, de teorías utópicas, irrealizables. La deliberación exige grupos pequeños, mucho más pequeños de los mínimos concebibles en la práctica política. Pues bien, ahí la ética y la bioética pueden resultar de enorme utilidad. La deliberación es el método clásico de la ética. Una de sus funciones sociales, quizá la principal, es la enseñanza y la práctica de la deliberación. De ahí su importancia en orden a lograr una verdadera democracia deliberativa. Sólo si las sociedades aprenden a deliberar, la democracia deliberativa podrá ser algún día realidad.

Quiero finalizar expresando mi convencimiento de que el éxito de la bioética se ha debido a la necesidad que la sociedad civil siente de reflexionar y deliberar sobre los 
problemas relativos a la gestión del medio ambiente, del cuerpo y de la vida de los seres humanos presentes, y de nuestros deberes para con las generaciones futuras. Ya no pueden ser los médicos, ni los políticos, ni los economistas, ni tampoco los sacerdotes o los teólogos quienes detenten el monopolio de la decisión en este tipo de cuestiones. Ha de ser la sociedad entera la que delibere y decida sobre ellas. Sólo de este modo se conseguirá lo que, por lo demás, todos consideramos imprescindible, el alumbramiento de un nuevo mundo más humano; es decir, de una nueva cultura.

\section{Referencias:}

1. Rawls J. A theory of justice. Cambridge, Mass: Belknap Press of Harvard University Press; 1971.

2. Nozick R. Anarchy, state, and utopia. New York : Basic Books; 1974.

3. Daniels N. Just health care. Cambridge New York: Cambridge University Press; 1985.

4. Daniels N. Am I my parents'keeper?: an essay on justice between the young and the old. New York: Oxford University Press; 1988.

5. Gutmann A. Democratic education. Princeton, N.J.: Princeton University Press; 1987. 